\title{
The Impact of an Intact Infrapatellar Fat Pad on Outcomes after Total Knee Arthroplasty
}

\author{
Leigh White, Nicholas Hartnell, Melissa Hennessy, and Judy Mullan \\ School of Medicine, University of Wollongong, Wollongong, NSW 2522, Australia \\ Correspondence should be addressed to Leigh White; lw844@uowmail.edu.au
}

Received 6 August 2015; Accepted 2 November 2015

Academic Editor: Doron Norman

Copyright ( 2015 Leigh White et al. This is an open access article distributed under the Creative Commons Attribution License, which permits unrestricted use, distribution, and reproduction in any medium, provided the original work is properly cited.

\begin{abstract}
Background. The infrapatellar fat pad (IPFP) is currently resected in approximately $88 \%$ of Total Knee Arthroplasties (TKAs). We hypothesised that an intact IPFP would improve outcomes after TKA. Methods. Patients with an intact IPFP participated in this cross-sectional study by completing two surveys, at 6 and 12 months after TKA. Both surveys included questions regarding kneeling, with the Oxford Knee Score also included at 12 months. Results. Sixty patients participated in this study. At 6 and 12 months, a similar number of patients were able to kneel, $40(66.7 \%)$ and $43(71.7 \%)$, respectively. Fifteen $(25.0 \%)$ patients were unable to kneel due to knee pain at 6 months; of these, nine (15\%) were unable to kneel at 12 months. Moreover, at 12 months, $90.0 \%$ of the patients reported minimal or no knee pain. There was no correlation between the inability to kneel and knee pain $(p=0.13)$. There was a significant correlation between the inability to kneel and reduced overall standardised knee function scores $(p=0.02)$. Conclusions. This was the first study to demonstrate improved kneeling and descending of stairs after TKA with IPFP preservation. These results in the context of current literature show that IPFP preservation reduces the incidence of knee pain 12 months after TKA.
\end{abstract}

\section{Introduction}

The strongest predictor of osteoarthritis (OA) is increasing age; thus with increasing life expectancy there is an increasing incidence of $\mathrm{OA}$ [1]. One of the most common joints to be effected by OA is the knee, which inevitably leads to severe pain and immobility [1]. Total Knee Arthroplasty (TKA) is proven to be a cost-effective treatment for endstage degenerative joint disorders including OA [2]. Each year the number of TKAs being performed continues to increase globally [3], predominately due to an increasing incidence of knee OA $[1,4-6]$.

Overall patient satisfaction with their TKA is very high, with as many as $81-89 \%$ of patients reporting that they are satisfied with their procedure [7-9]. Areas of highest satisfaction, among patients receiving TKAs, include improved knee stability, reduced pain after long periods of sitting, and the ability to complete basic activities of daily living, inclusive of bathing [7-10]. Despite the high level of patient satisfaction, there are also areas of patient dissatisfaction which need to be considered. The most common reasons for patient dissatisfaction include pain resulting from the procedure, an inability to kneel, and trouble descending stairs following the procedure [9]. Given that the number of patients undergoing TKA is increasing, it is important to explore strategies which could be used to improve patient satisfaction in these areas $[8,9]$. One such strategy, currently being investigated, is to keep the infrapatellar fat pad (IPFP) intact during routine TKA. To date, there is a paucity of evidence regarding the post-TKA outcomes when the IPFP has not been removed during the procedure [11].

The IPFP has been traditionally removed in order to enhance surgical access during TKA [12]. Over the past few decades, some of the equipment required to perform a TKA has evolved and changed significantly, such as the tibial alignment guides. These guides have become much smaller and subsequently can be used without the need to resect and remove the IPFP for surgical access. However, even with the improvement and the reduction in size of these alignment guides, current literature indicates that the IPFP is still being partially or completely removed in up to $88 \%$ of TKAs [9].

Emerging evidence suggests that the IPFP may play an important role in reducing postoperative anterior knee pain 
TABle 1: The Oxford Knee Score [28, 29].

\begin{tabular}{ll}
\hline Question number & Question \\
\hline 1 & Describe the pain you usually have from your knee? (Pain) \\
2 & How much trouble do you have washing and drying yourself (all over) because of your knee (Function) \\
3 & How much trouble do you have getting in/out of your car or using public transport because of your knee? (Function) \\
4 & For how long have you been able to walk before pain from your knee becomes severe? (with or without a stick) (Pain) \\
5 & After a meal (sat at a table), how painful has it been for you to stand up from a chair because of your knee? (Pain) \\
6 & Have you been limping when walking, because of your knee? (Function) \\
7 & Could you kneel down and get up again afterwards? (Function) \\
8 & Have you been troubled by pain from your knee in bed at night? (Pain) \\
9 & How much has pain from your knee interfered with your usual work (including housework)? (Pain) \\
10 & Have you felt that your knee might suddenly "give way" or let you down? (Function) \\
11 & Could you do household shopping on your own? (Function) \\
12 & Could you walk down one flight of stairs? (Function) \\
\hline
\end{tabular}

following TKA [13-17]. Even though Maculé et al. [18] and Tanaka et al. [19] found that patients with an intact IPFP experienced the same or higher rates of knee pain in the short term (less than six months after TKA), other studies that considered the long-term effects (beyond six months) showed that patients with an intact IPFP experienced less pain than those with an IPFP resection after TKA $[12,19,20]$.

In addition to the potential impact of IPFP resection on anterior knee pain after TKA, it may also have an impact on patients' ability to kneel. TKA alone can improve patients' ability to kneel, from approximately $2-4 \%$ prior to the procedure $[21,22]$ to between 41 and $73 \%$ following the procedure $[9,23-26]$. Notably, however, some of these patients will experience some degree of difficulty with kneeling following the procedure $[21,23,25]$. This level of difficulty is important, because kneeling is a function that many people require in order to successfully perform everyday tasks, such as professional duties (e.g., carpet laying and plumbing) and recreational activities (e.g., gardening and playing lawn bowls) [21]. The ability to kneel holds an even greater level of importance in middle eastern and far eastern cultures where kneeling is integral to everyday activities, such as praying and sitting for meals [22]. Of all functional outcomes kneeling has been shown to have the least amount of improvement following a TKA [27]. It is therefore surprising that only a small number of studies have looked at kneeling after TKA [22-25] and that no studies have looked at the impact of an intact IPFP on kneeling. The aim of this study was to investigate the impact of an intact IPFP after TKA on knee pain and knee functions, such as the ability to kneel and descend stairs.

\section{Material and Methods}

2.1. Overview of Study Design. Following ethics approval from the University of Wollongong human research ethics committee, patients attending an outpatient clinic between July 2013 and May 2014, who did not have their IPFP removed during their routine TKA, were invited to participate in this cross-sectional study. Volunteering patient participants were asked to complete two surveys, which were mailed out to them at 6 and 12 months after TKA. Both surveys included demographic questions and kneeling survey questions, with the addition of the validated Oxford Knee Score [28, 29] in the 12-month survey. These procedures were all performed by the same surgeon at two different hospitals using a cemented, hydroxyapatite-coated posterior cruciate retaining prosthesis incorporated into their tibia. Each knee replacement included patellar resurfacing with a cemented polyethylene button. Surgical access for each operation was gained via a midline incision, with a medial parapatellar approach.

2.2. Kneeling Survey Questions. The four questions in both the 6-month and 12-month surveys which focused on kneeling included the following: (1) Are you able to kneel on your replaced knee? (2) Do you have pain with kneeling? (3) Do you have discomfort or increased pressure within the knee with kneeling? (4) Does pain stop/prevent you from kneeling. These questions were scored as yes or no answers and each question was analysed using descriptive statistical analysis.

2.3. Oxford Knee Score $[28,29]$. In addition to the kneeling survey questions discussed above, at twelve months patients were also asked to complete validated and reliable Oxford Knee Score (OKS) [28, 29] as part of their 12-month postprocedure questionnaire. The $\mathrm{OKS}$ is a standardised questionnaire consisting of twelve questions, which is used to gauge overall knee pain and knee function after TKA, including the ability to descend stairs and kneel $[28,29]$ (Table 1).

Patients were asked to base their responses to the OKS on their experiences over the previous four weeks. The possible responses were given a numerical value from zero (worst) to four (best). Overall pain and function scores were calculated based on responses to the OKS [9]. The pain score was calculated by adding together the scores of the five questions related to pain (questions 1, 4, 5, 8, and 9; Table 1), an approach used by Baker et al. [9]. The overall function score was calculated by adding together the scores of the function based questions (questions 2, 3, 6, 7, 10, 11, and 12; Table 1) [9]. The overall pain and function scores were converted to standardised scores to allow for comparison. This was done 
TABLE 2: Results of kneeling survey at 6 and 12 months after Total Knee Arthroplasty.

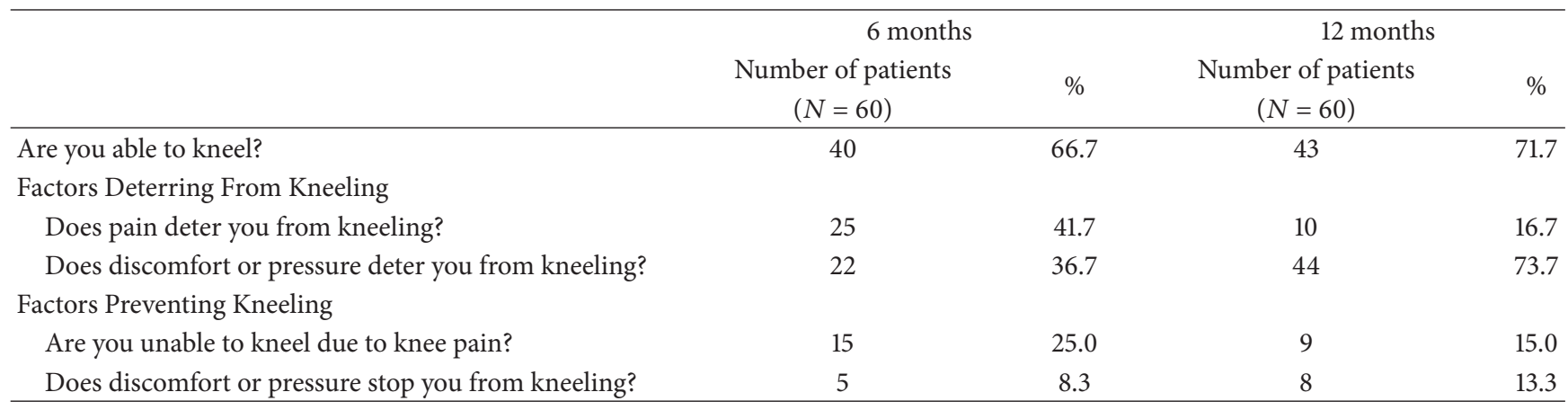

by dividing the overall scores by the highest possible score for pain (20) or function (28), a technique used by other researchers in the literature [9]. For example, a pain score of 15 produced a standardised pain score of $0.75(15 / 20=0.75)$ and a function score of 14 produced a standardised function score of $0.50(14 / 28=0.50)$. The standardised scores were then grouped together to compare the overall pain and function of the patients that could kneel versus those who could not.

2.4. Statistical Analysis. The data was analysed using the statistics function of Microsoft Excel 2010. A multivariate logistic regression of the standardised knee pain and knee function scores were conducted to provide comparisons between the group of patients that could kneel and those that could not. A $p$ value $\leq 0.05$ was considered to be statistically significant.

\section{Results}

3.1. Patient Demographic Data. Seventy-nine eligible patients were mailed questionnaires at six and twelve months after TKA. Sixty patients returned both questionnaires (32 female, 28 male), indicating a response rate of $75.9 \%$. The mean age of the patients at 6 and 12 months was 67 years.

3.2. Patient Kneeling Capabilities. At six months after TKA, 40 of the 60 respondents $(66.7 \%)$ were able to kneel. Of the 20 patients unable to kneel, $5(8.3 \%)$ reported that this was due to discomfort or knee pressure (Table 2). The remaining $15(25 \%)$ reported that they were unable to kneel due to knee pain (Table 2). Pain was reported as a deterrent factor from kneeling in $41.7 \%(n=25)$ of those surveyed (Table 2$)$. Of the 60 respondents surveyed at 12 months after TKA, 43 $(71.7 \%)$ reported that they were able to kneel. A total of 9 (15\%) patients were unable to kneel due to pain, and 8 (13.3\%) were unable to kneel due to discomfort and knee pressure (Table 2).

3.3. Overall Oxford Knee Score Results. At 12 months after TKA, the average OKS for the 60 patient participants was $42.7 / 48$ (Figure 1). The overall OKS result is a validated indicator of how successful a TKA has been for the patient $[28,29]$. The overall OKS can range from zero, which is an

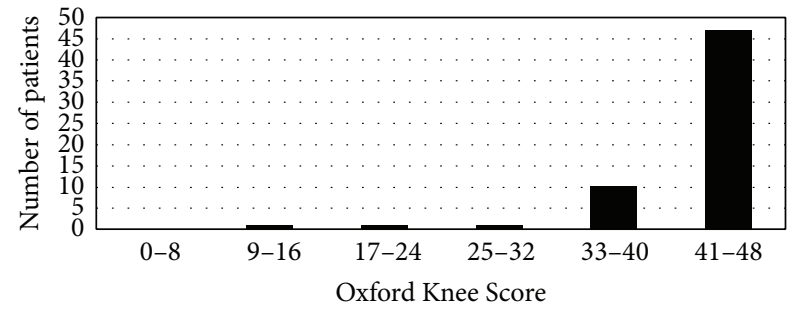

FIgure 1: Overall Oxford Knee Score distribution in 60 patients at 12 months after Total Knee Arthroplasty.

TAble 3: Frequency (\%) of responses to the Oxford Knee Score questions for stair-descending, knee stability, and kneeling ability at 12 months after Total Knee Arthroplasty $(n=60)$.

\begin{tabular}{lccccc}
\hline & 0 (worst) & 1 & 2 & 3 & 4 (best) \\
\hline $\begin{array}{l}\text { Ability } \\
\text { with stairs }\end{array}$ & $0(0.0)$ & $1(1.7)$ & $5(8.3)$ & $15(25.0)$ & $39(65.0)$ \\
\hline $\begin{array}{l}\text { Knee } \\
\text { stability }\end{array}$ & $0(0.0)$ & $0(0.0)$ & $1(1.7)$ & $9(15.0)$ & $50(83.3)$ \\
\hline $\begin{array}{l}\text { Kneeling } \\
\text { ability }\end{array}$ & $5(8.3)$ & $5(8.3)$ & $16(26.7)$ & $20(33.3)$ & $14(23.3)$ \\
\hline
\end{tabular}

extremely poor result, to 48 , which represents a knee with perfect function and no pain $[28,29]$. In this study, the lowest overall OKS recorded was 13 , which means that they scored on average one to two out of four for each question. In contrast, however, 90 percent of patients $(n=54)$ recorded a score of 36 or higher, which indicates that they had minimal to no problems with the TKA [27] (Figure 1).

3.3.1. Ability to Descend Stairs, Knee Stability, Kneeling, and Overall Knee Function from the OKS. At 12 months after TKA, the majority of patients reported on the OKS that they were able to descend stairs with minimal to no difficulty (90\%), with a mean score of 3.5 out of a possible score of 4 for ability with stairs. Patients had minimal to no problems with their knee stability (3.8/4) and overall knee function $(24.8 / 28)$. Kneeling was the only question where patients reported mild to moderate difficulty, with a mean score of 2.6 out of 4 . The frequency of responses to each of these questions is featured in Table 3. 
TABLE 4: Standardised pain and function scores in patients that could and could not kneel at 12 months after Total Knee Arthroplasty.

\begin{tabular}{lcccccc}
\hline Score $^{*}$ & & Overall & & Able to kneel & & \multicolumn{2}{c}{$\begin{array}{c}\text { Unable to kneel } \\
\text { Standardised score (95\% CI) }\end{array}$} \\
\hline Pain & 60 & $0.90(0.86$ to 0.94$)$ & 43 & $0.91(0.87 \text { to } 0.95)^{\#}$ & 17 & $0.85(0.74$ to 0.96$)$ \\
Function & 60 & $0.89(0.86$ to 0.92$)$ & 43 & $0.91(0.88 \text { to } 0.94)^{¥}$ & 17 & $0.83(0.74$ to 0.90$)$ \\
\hline
\end{tabular}

${ }^{*}$ Pain is the summation of questions $1,4,5,8$, and 9 of the OKS; function is the summation of questions $2,3,6,7,10,11$, and 12 of the OKS. ${ }^{*}$ There was a nonsignificant difference between the pain scores of the patients that could kneel and those that could not $(p=0.13) .{ }^{¥}$ There was a significant difference in function scores between the group of patients that can kneel and those who cannot $(p=0.02)$.

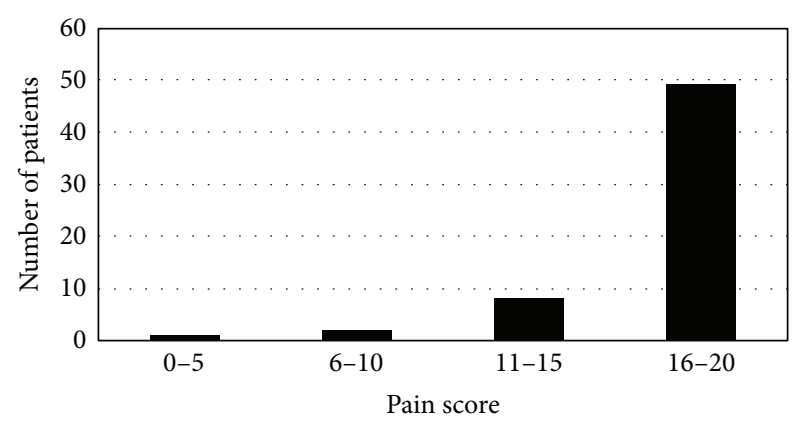

FIGURE 2: Postoperative pain scores derived from questions 1, 4, 5, 8 , and 9 of the Oxford Knee Score at 12 months after Total Knee Arthroplasty $(n=60)$.

3.3.2. Knee Pain at 12 Months after TKA from the OKS. The mean pain score derived from the OKS was 17.9/20. Figure 2 shows the pain scores of each patient as calculated from the OKS. The majority of patients (90\%) had overall pain scores between 15 and 20, indicating that they experience minimal to no pain at 12 months after TKA. Only six (10\%) patients reported their overall knee pain to be in the mild to moderate range (10-14) (Figure 2).

3.4. Analysis of Ability to Kneel in Relation to Standardised Pain and Function Scores. The overall group of patients $(n=$ 60 ) had a mean standardised pain score of 0.90 and mean standardised function score of 0.89 (Table 4 ). There was a significant difference in the standardised function scores between the patients that could kneel (0.91) and those that could not (0.82), as determined by multivariate regression modeling $(p=0.02)$. There was also a difference in the standardised pain score between those who could kneel (0.91) and those who could not (0.85). However, this difference was not significant $(p=0.13)$.

\section{Discussion}

Results of this study showed that a high proportion of patients with an intact IPFP after TKA could kneel at 6 and 12 months following the procedure $(66.7 \%$ and $71.7 \%$, resp.). At 12 months after TKA, $90.0 \%$ of patients reported minimal to no knee pain and that they had minimal to no difficulty descending stairs. Kneeling was the only question where patients reported mild to moderate difficulty (2.6/4). Of comparisons between patients who could and could not kneel at 12 months after TKA, it was found that the inability to kneel was significantly related to knee function $(p=0.02)$ rather than knee pain.

4.1. Knee Pain. At 12 months after TKA, $90 \%$ of patients reported their knee pain to be mild or nonexistent. The low incidence of knee pain shown in this current study agrees with the results of a number of other studies, which have shown a significant reduction in the incidence of knee pain beyond six months after TKA in patients with an intact IPFP $[12,19,20]$. The incidence of knee pain in the IPFP resection after TKA increased to almost twice that of the preservation group at 5.1 years after procedure [12].

The overall standardised pain score calculated in our study was 0.90 (0.86 to $0.94 ; 95 \%$ CI). Baker et al. (2007) conducted a study of 8,231 patients that had very similar demographics and used an almost identical surgical technique to our study [9]. They found that $84.1 \%$ of their patients had either a partial or complete IPFP resection which yielded standardised pain scores of 0.81 ( 0.81 to $0.82 ; 95 \% \mathrm{CI})$, which was a lower result than our findings. From this comparison of our study with the current literature, it could be proposed that an intact IPFP contributes to reduced medium to long-term knee pain after TKA. One of the most likely explanations may be that the IPFP mediates pain and inflammation while there is an arthritic, inflammatory process occurring in the knee joint $[30,31]$. Therefore, once the inflammation in the joint is resolved through TKA, the stimulus for the IPFP to mediate pain and inflammation may no longer be present.

4.2. Ability to Descend Stairs. To our knowledge, this was the first study to examine the ability to descend stairs in patients with an intact IPFP after TKA. Hassaballa et al. completed the only previous study to investigate the impact of TKA on the patients' ability to descend stairs [22]. They found that $73.1 \%$ of patients were able to descend stairs with minimal or no difficulty at 12 months after TKA; however, their methodology did not disclose the rate of IPFP resections conducted as part of the study [22]. As removal of the IPFP was common practice in the United Kingdom during the time of Hassaballa et al.s study, it is likely that resection occurred in the majority of these patients [9]. In our study, which looked exclusively at patients with an intact IPFP after TKA, it was found that $90.0 \%$ of the patients could descend stairs with minimal to no difficulty at 12 months following their procedure. This is an increase of approximately $17 \%$ from Hassaballa et al's study, which could suggest that an intact IPFP after TKA increases the patient's ability to descend stairs [22]. One of the reasons we believe that an intact IPFP 
may increase the patient's ability to descend stairs is due to the effect that resection has on the patellar tendon. Recent studies have shown that IPFP resection causes shortening of the patellar tendon, which causes a reduction in the range of motion (ROM) in the knee joint $[11,19,32]$. This is important because a reduction in knee ROM impairs patients' ability to descend stairs [33].

4.3. Ability to Kneel. Kneeling is a crucial knee function to many people around the world and is currently a functional outcome that shows the least amount of improvement following routine TKA procedures [21, 22, 27]. An important function of the IPFP is to cushion the interface between patellar tendon and the tibial plateau, especially during kneeling [34]. Of the small number of studies that have investigated the impact of TKA on the ability to kneel, only one has explicitly stated the rate of IPFP resection. Baker et al. investigated the ability to kneel in 8,231 patients who had TKA, with $84.1 \%$ of these subjects having the IPFP resected [9]. Results of Baker et al's study showed that $57 \%$ of patients found it extremely difficult to impossible to kneel at 12 months after TKA [9]. In contrast, our study, which exclusively looked at patients with an intact IPFP, found that only $16.6 \%$ of patients found it extremely difficult to impossible to kneel at 12 months following the procedure. This suggests that having an intact IPFP may increase the likelihood that a patient can kneel following TKA.

There are commonly a number of reasons why people are unable to kneel after TKA, including pain, skin hypoesthesia, and decreased ROM [22]. In order to gain a better understanding of why the patients in our study were unable to kneel, we divided the results of those that could and could not kneel for further analysis. In these groupings we then compared standardised pain and function scores. Through this analysis we found that those that could kneel had a significantly higher standardised function score $(p=0.02)$ than those who could not. However, we found no significant difference between groups in standardised pain scores $(p=$ $0.13)$. Therefore, these results show that pain is unlikely to be a limiting factor in the ability to kneel in post-TKA patients with an intact IPFP. We believe that the IPFP reduces pain during kneeling as it acts as a cushion which protects the patellar tendon from pressing against the metal tibial plateau prosthesis.

Limitations. As this was a pilot study conducted in a limited time frame, several limitations need to be acknowledged. This study did not include a control group, did not look at preoperative scores, only included a small sample size, and only investigated patients treated by one surgeon in two hospitals. A randomised controlled trial (RCT) with preoperative scoring and patients treated by multiple surgeons would have provided much more robust and clinically applicable results. An RCT would have also allowed for a higher level comparison of the impact of an intact versus resected IPFP.

In conclusion, our results in combination with the evidence in the current literature showed that IPFP preservation may lead to more favourable outcomes after TKA in OA patients. We found a correlation between an intact IPFP and reduced rates of knee pain at 12 months after TKA. This is the first study to look at the impact of an intact IPFP on functional outcomes such as kneeling and descending stairs after TKA. The results of this study indicate that an intact IPFP may improve the ability to kneel and descend stairs after TKA. The results of this study are not substantial enough to justify new guidelines to state that the IPFP should be left in situ during TKA. For this reason a well-constructed RCT needs to be conducted.

\section{Ethical Approval}

Authorisation to perform this study was given by a local committee and was performed in accordance with the Ethical Standards of the 1964 Declaration of Helsinki as revised in 2000.

\section{Consent}

All patients gave informed consent prior to enrolling in the study.

\section{Conflict of Interests}

The authors declare that they have no conflict of interests (both personal and financial).

\section{References}

[1] N. Arden and M. C. Nevitt, "Osteoarthritis: epidemiology," Best Practice \& Research Clinical Rheumatology, vol. 20, no. 1, pp. 325, 2006.

[2] NSW Agency for Clinical Innovation (NSW ACI), Musculoskeletal Network: NSW Evidence Review Preoperative, Perioperative and Postoperative Care of Elective Primary Total Hip and Knee Replacement, Agency for Clinical Innovation, Chatswood, Australia, 2012, http://www.aci.health.nsw.gov.au/_data/assets/ pdf_file/0020/172091/EJR-Evidence-Review.PDF.

[3] S. M. Kurtz, K. L. Ong, E. Lau et al., "International survey of primary and revision total knee replacement," International Orthopaedics, vol. 35, no. 12, pp. 1783-1789, 2011.

[4] C. J. Lavernia, J. F. Guzman, and A. Gachupin-Garcia, "Cost Effectiveness and quality of life in knee arthroplasty," Clinical Orthopaedics and Related Research, vol. 345, pp. 134-139, 1997.

[5] Australian Orthopaedic Association (AOA), "Hip and Knee Arthroplasty: Annual Report 2013," National Joint Replacement Registry, 2013, https://aoanjrr.dmac.adelaide.edu.au/presentations-2013.

[6] L. M. March and H. Bagga, "Epidemiology of osteoarthritis in Australia," Medical Journal of Australia, vol. 180, no. 5, supplement, pp. S6-S10, 2004.

[7] O. Robertsson, M. Dunbar, T. Pehrsson, K. Knutson, and L. Lidgren, "Patient satisfaction after knee arthroplasty: a report on 27,372 knees operated on between 1981 and 1995 in Sweden," Acta Orthopaedica Scandinavica, vol. 71, no. 3, pp. 262-267, 2000.

[8] J. G. Anderson, R. L. Wixson, D. Tsai, S. D. Stulberg, and R. W. Chang, "Functional outcome and patient satisfaction in total knee patients over the age of 75," Journal of Arthroplasty, vol. 11, no. 7, pp. 831-840, 1996. 
[9] P. N. Baker, J. H. van der Meulen, J. Lewsey, and P. J. Gregg, “The role of pain and function in determining patient satisfaction after total knee replacement. Data from the national joint registry for England and Wales," The Journal of Bone \& Joint Surgery-British Volume, vol. 89, no. 7, pp. 893-900, 2007.

[10] D. A. Heck, R. L. Robinson, C. M. Patridge, R. M. Lubitz, and D. A. Feund, "Patient outcomes after knee replacement," Clinical Orthopaedics and Related Research, vol. 356, pp. 93-110, 1998.

[11] A. Van Beeck, S. Clockaerts, J. Somville et al., "Does infrapatellar fat pad resection in total knee arthroplasty impair clinical outcome? A systematic review," The Knee, vol. 20, no. 4, pp. 226231, 2013.

[12] P. Pinsornsak, K. Naratrikun, and S. Chumchuen, "The effect of infrapatellar fat pad excision on complications after minimally invasive TKA: a randomized controlled trial," Clinical Orthopaedics and Related Research, vol. 472, no. 2, pp. 695-701, 2014.

[13] N. Popovic and R. Lemaire, "Anterior knee pain with a posterior-stabilized mobile-bearing knee prosthesis: the effect of femoral component design," The Journal of Arthroplasty, vol. 18, no. 4, pp. 396-400, 2003.

[14] T. S. Waters and G. Bentley, "Patellar resurfacing in total knee arthroplasty: a prospective, randomized study," The Journal of Bone \& Joint Surgery-American Volume, vol. 85, no. 2, pp. 212217, 2003.

[15] D. J. Wood, A. J. Smith, D. Collopy, B. White, B. Brankov, and M. K. Bulsara, "Patellar resurfacing in total knee arthroplasty: a prospective, randomized trial," The Journal of Bone \& Joint Surgery-American Volume, vol. 84, no. 2, pp. 187-193, 2002.

[16] R. S. J. Burnett, J. L. Boone, K. P. McCarthy, S. Rosenzweig, and R. L. Barrack, "A prospective randomized clinical trial of patellar resurfacing and nonresurfacing in bilateral TKA," Clinical Orthopaedics and Related Research, vol. 464, pp. 65-72, 2007.

[17] A. J. Smith, D. J. Wood, and M.-G. Li, “Total knee replacement with and without patellar resurfacing: a prospective, randomised trial using the Profix total knee system," The Journal of Bone \& Joint Surgery - British Volume, vol. 90, no. 1, pp. 43-49, 2008.

[18] F. Maculé, S. Sastre, S. Lasurt, P. Sala, J.-M. Segur, and C. Mallofré, "Hoffa's fat pad resection in total knee arthroplasty," Acta Orthopaedica Belgica, vol. 71, no. 6, pp. 714-717, 2005.

[19] N. Tanaka, H. Sakahashi, E. Sato, K. Hirose, and T. Isima, "Influence of the infrapatellar fat pad resection in a synovectomy during total knee arthroplasty in patients with rheumatoid arthritis," Journal of Arthroplasty, vol. 18, no. 7, pp. 897-902, 2003.

[20] R. M. Meneghini, J. L. Pierson, D. Bagsby, M. E. Berend, M. A. Ritter, and J. B. Meding, "The effect of retropatellar fat pad excision on patellar tendon contracture and functional outcomes after total knee arthroplasty," The Journal of Arthroplasty, vol. 22, no. 6, pp. 47-50, 2007.

[21] J. Dawson, R. Fitzpatrick, D. Murray, and A. Carr, "Questionnaire on the perceptions of patients about total knee replacement," The Journal of Bone \& Joint Surgery-British Volume, vol. 80, no. 1, pp. 63-69, 1998.

[22] M. A. Hassaballa, A. J. Porteous, and I. D. Learmonth, "Functional outcomes after different types of knee arthroplasty: kneeling ability versus descending stairs," Medical Science Monitor, vol. 13, no. 2, pp. CR77-CR81, 2007.
[23] M. A. Hassaballa, A. J. Porteous, J. H. Newman, and C. A. Rogers, "Can knees kneel? Kneeling ability after total, unicompartmental and patellofemoral knee arthroplasty," The Knee, vol. 10, no. 2, pp. 155-160, 2003.

[24] M. Mardani Kivi, M. Karimi Mobarakeh, K. Hashemi Motlagh, and K. Saheb Ekhtiari, "Evaluation of kneeling ability after total knee replacement in patients with osteoarthritis of the knee," Minerva Ortopedica e Traumatologica, vol. 63, no. 3, pp. 177-184, 2012.

[25] S. H. Palmer, C. T. Servant, J. Maguire, E. N. Parish, and M. J. Cross, "Ability to kneel after total knee replacement," The Journal of Bone \& Joint Surgery -British Volume, vol. 84, no. 2, pp. 220-222, 2002.

[26] P. F. Sharkey and A. J. Miller, "Noise, numbness, and kneeling difficulties after total knee arthroplasty. Is the outcome affected?" Journal of Arthroplasty, vol. 26, no. 8, pp. 1427-1431, 2011.

[27] D. P. Williams, C. M. Blakey, S. G. Hadfield, D. W. Murray, A. J. Price, and R. E. Field, "Long-term trends in the Oxford knee score following total knee replacement," The Journal of Bone \& Joint Surgery -British Volume, vol. 95, no. 1, pp. 45-51, 2013.

[28] D. W. Murray, R. Fitzpatrick, K. Rogers et al., "The use of the Oxford hip and knee scores," The Journal of Bone \& Joint Surgery-British Volume, vol. 89, no. 8, pp. 1010-1014, 2007.

[29] K. I. A. Reddy, L. R. Johnston, W. Wang, and R. J. Abboud, “Does the oxford knee score complement, concur, or contradict the american knee society score?" Journal of Arthroplasty, vol. 26, no. 5, pp. 714-720, 2011.

[30] É. Toussirot, G. Streit, and D. Wendling, “The contribution of adipose tissue and adipokines to inflammation in joint diseases," Current Medicinal Chemistry, vol. 14, no. 10, pp. 10951100, 2007.

[31] H. Dumond, N. Presle, B. Terlain et al., "Evidence for a key role of leptin in osteoarthritis," Arthritis and Rheumatism, vol. 48, no. 11, pp. 3118-3129, 2003.

[32] M. Bohnsack, A. Wilharm, C. Hurschler, O. Rühmann, C. Stukenborg-Colsman, and C. J. Wirth, "Biomechanical and kinematic influences of a total infrapatellar fat pad resection on the knee," The American Journal of Sports Medicine, vol. 32, no. 8, pp. 1873-1880, 2004.

[33] N. Shah, "Clinical suggestion: increasing knee range of motion using a unique sustained motion," North American Journal of Sports Physical Therapy, vol. 3, pp. 110-113, 2008.

[34] G. Hamarneh, V. Chu, M. Bordalo-Rodrigues, and M. Schweitzer, "Deformation analysis of Hoffa's fat pad from CT images of knee flexion and extension," in Medical Imaging 2005: Physiology, Function, and Structure from Medical Images, vol. 5746 of Proceedings of SPIE, pp. 703-710, April 2005. 


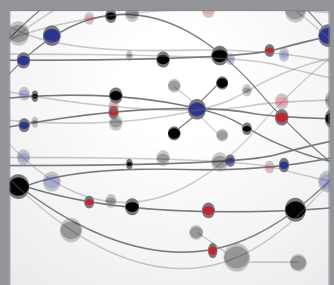

The Scientific World Journal
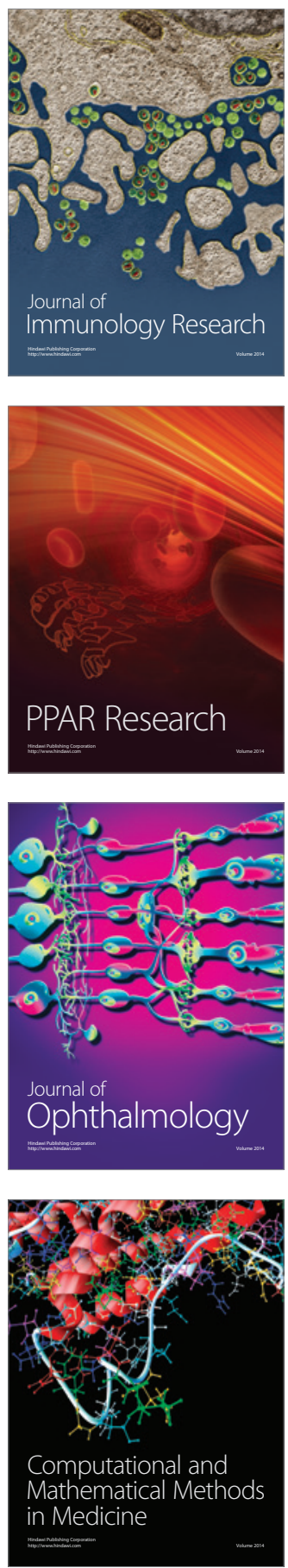

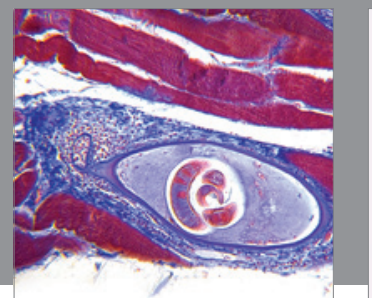

Gastroenterology

Research and Practice
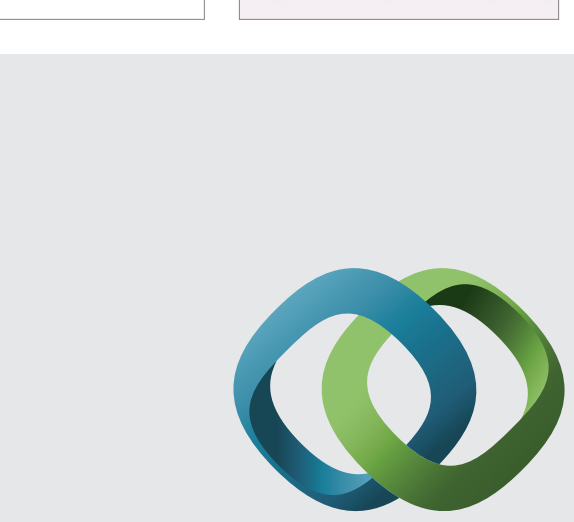

\section{Hindawi}

Submit your manuscripts at

http://www.hindawi.com
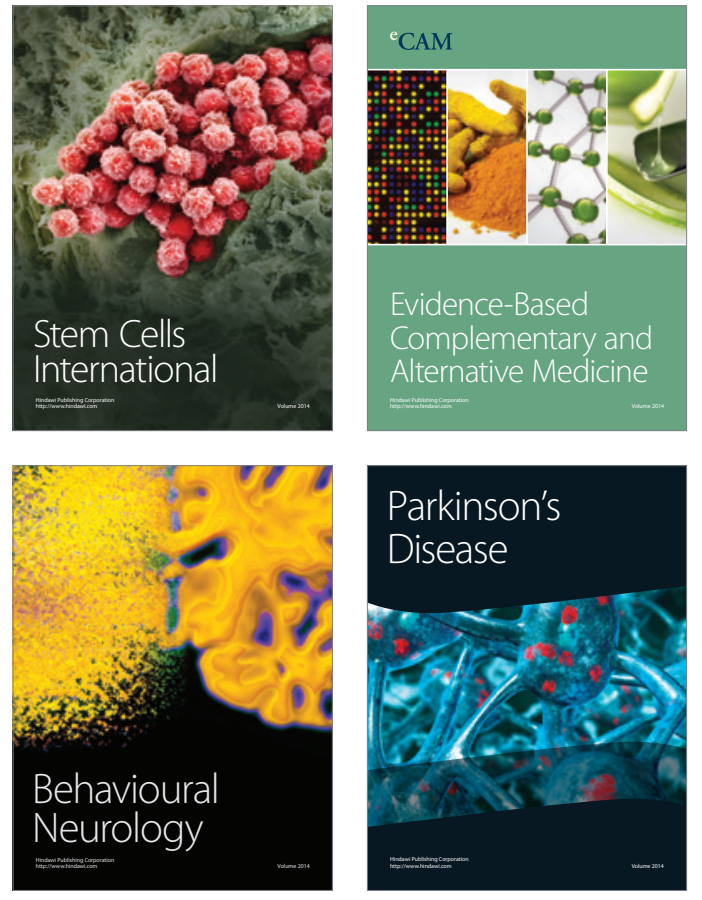
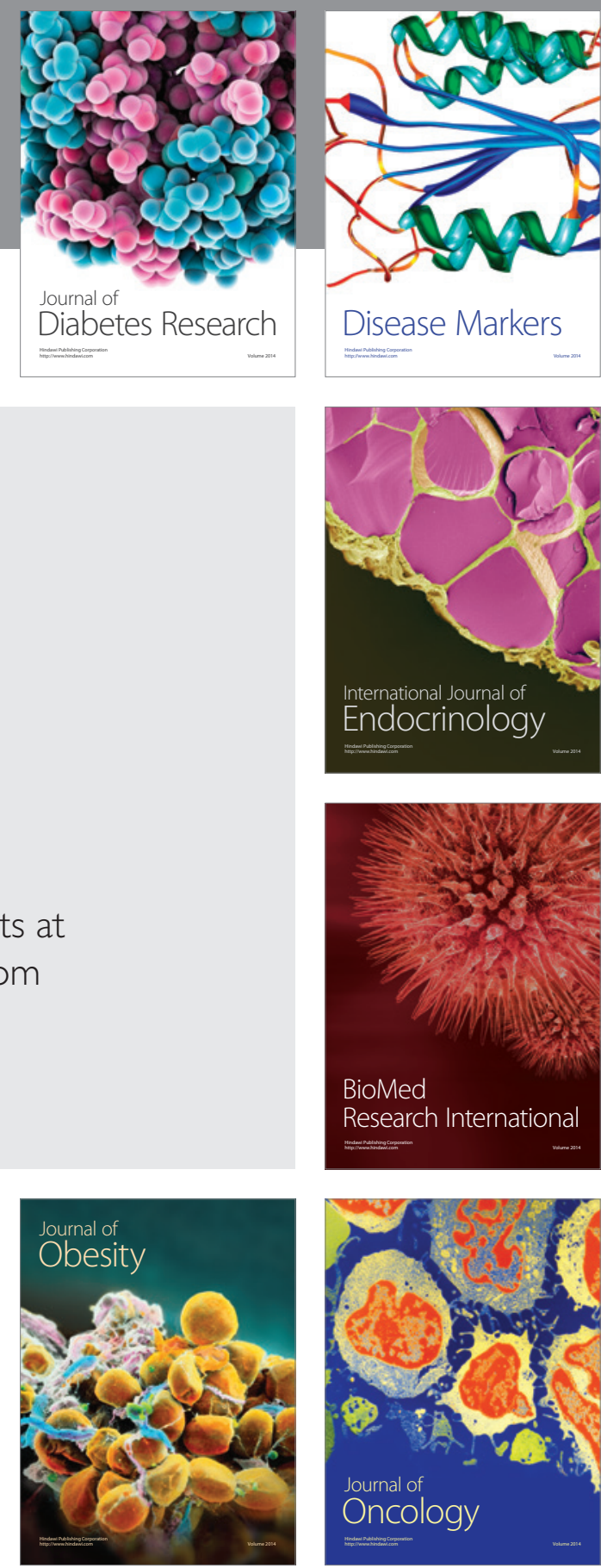

Disease Markers
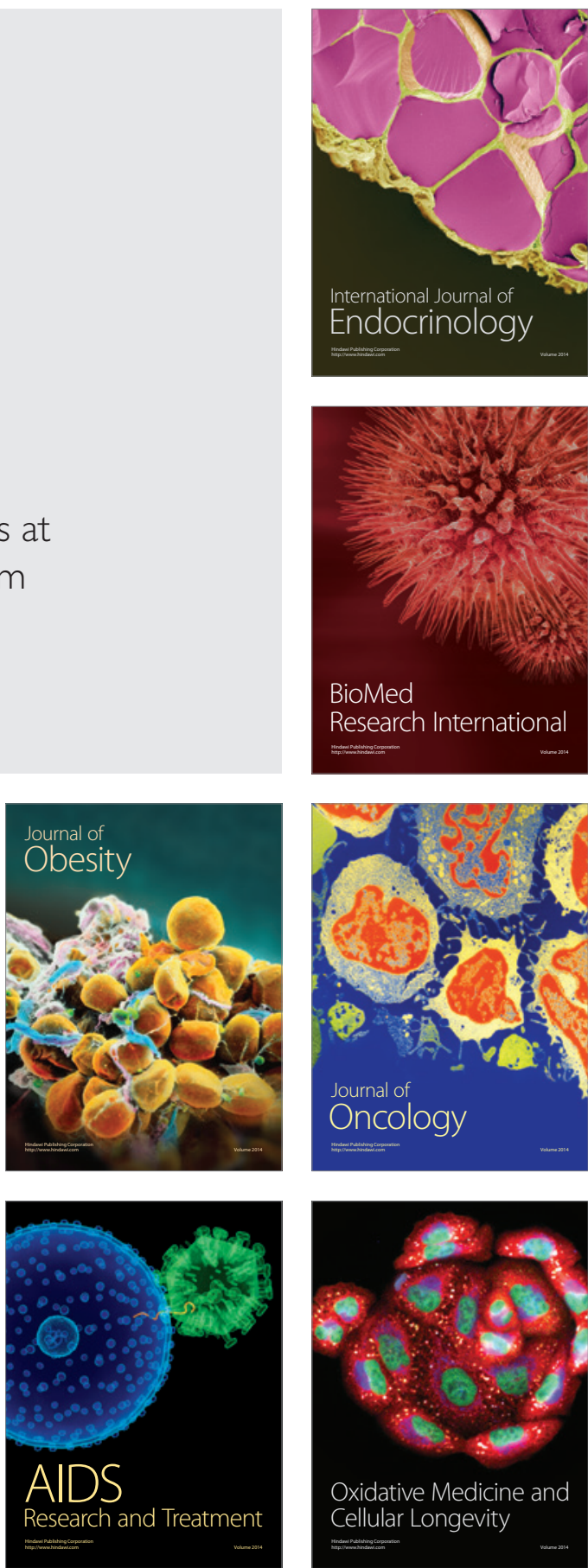